\title{
Habilidades Sociales Según Sexo y Especialidad, en Estudiantes de la Facultad de Ciencias de la Educación de la Universidad Nacional Jorge Basadre Grohmann, Tacna - 2008
}

RESPONSABt.E: Mgr. Alberto Lanchipa Ale

RESUMEN. Hoy en dia, resulta importante el favorecer el contacto positivo entre los miembros de la comunidad, ya que las interacciones afectuosas, abiertas, empáticas y confiadas promueven ambientes saludables, tan necesarios para un óptimo desarrollo de la personalidad.

El presente trabajo de investigación llegó a establecer que no existen diferencias significativas en las habilidades sociales según sexo y especialidad en los estudiantes de la Facultad de Ciencias de la Educación (FACE) de la Universidad Nacional Jorge Basadre Grohmann (UNJBG). Frente a ello es necesario considerar en el curriculo, asignaturas $y$ contenidos que permitan que los estudiantes adquieran habilidades sociales que optimicen sus relaciones interpersonales.
MIE virros: Dr: Elmer Rivera Mansilla

Lic: Luisa Esther Llanos Sucapuca

\begin{abstract}
Today in day, it is important favoring the positive contact among the members of the community, since the affectionate, open interactions, empaticas and confident they promote healthy atmospheres, so necessary for a good development of the personality.
\end{abstract}

The present investigation work ends up settling down that it doesn't exist significant differences in the social abilities according to sex and specialty in the students of the Ability of Sciences of the Education, in front of it is necessary to consider in the curricula of studies, subjects and contents that allow the students to acquire social abilities that optimize their interpersonal relationships.
INTRODUCCIÓN Conocedores que la función principal del docente debe de ser la de formador de nuevas generaciones, en el actual sistema educativo, este debe de asumir un nuevo rol, muy diferente al que estamos acostumbrados. Además de transmitir información o sus conocimientos debe de promover en sus alumnos habilidades sociales que le permitan crecer como personas y adaptarse a los diferentes cambios y roles que asumirá en la sociedad.

Sin embargo, se observa que no se pone énfasis en la educación, en la personalidad que debe poseer el futuro educador, así como en el formador. Observamos que no se modelan conductas que fortalezcan la adquisición de habilidades sociales, siendo por ello necesario que en la estructura curricular se inserten contenidos que promuevan aprendizajes de competencias sociales.

Es a partir de lo planteado, que la presente investigación, busca identificar el nivel de habilidades sociales según sexo y especialidad, que presentan los estudiantes de la Facultad de Ciencias de la Educación que nos permita visualizar cúal es el logro que ofrecen y qué es lo que se espera de ellos como futuros formadores.

Frecuentemente observamos en nuestro contexto universitario el comportamiento que reflejan nuestros estudiantes en las relaciones entre grupos, como resultado de ello, se hace evidente, enormes diferencias en cuanto a sus habilidades sociales, muchas de las cuales promueven una fácil integración que propicia una fácil y exitosa comunicación y en otros casos se hace notoria una conducta retraida y aislada.

La adquisición y el modelamiento de conductas sociales proactivas, en estudiantes que se forman para desarrollar actividades de carácter pedagógico, en tiempos actuales, resulta de vital importancia, ya que el profesional en educación deberá interactuar con personas de diferente edad, sexo y condición socioeconómica, y para ello serán necesarios que este posea, como parte de su repertorio conductual, un conjunto de conductas que favorezcan y promuevan el manejo de óptimas relaciones interpersonales.

La adquisición y el manejo de habilidades sociales se hacen necesarios e importantes, ya que estas favorecen el contacto entre los miembros de una comunidad, facilitando interacciones afectuosas, abiertas, empáticas y confiadas. Asi mismo, se facilita la adquisición de experiencias para el logro de aprendizajes significativos, ya que los estudiantes con estas cualidades se muestran más activos e interactivos en la construcción de sus propios saberes, se motivan académicamente y mejoran sus habilidades para el conocimiento, obteniendo de este modo un mayor rendimiento académico.

A lo señalado se agrega, que la educación actual y las perspectivas de su desarrollo exigen un replanteamiento de las funciones del docente, de ahí que hoy en día se pone énfasis en la personalidad y en la formación de esta, ya que la labor pedagógica no solo se limita a la posesión de conocimientos, ya que el docente, como ser humano, funciona como un todo integrado. 
Considerando todo lo anteriormente mencionado nos planteamos la siguiente interrogante: ¿Qué nivel de habilidades sociales según sexo y especialidad presentan los estudiantes de la Facultad de Ciencias de la Educación de la UNJBG?

Las habilidades sociales no son simples normas de urbanidad impuestas por la sociedad e internalizadas por cada uno de nosotros. Tampoco son un código de sabiduría, que nos enseña cómo actuar en cada ocasión, son conductas verbales y no verbales que facilitan una relación asertiva, que se manifiesta de forma externa y de modo estable, es decir, que la persona actúe normalmente así, aunque tenga algún fallo esporádico.

Decimos que son conductas verbales, porque la mayor parte de nuestra comunicación se hace con palabras. No solo se debe utilizar palabras expresivas y correctas sino decirlas con el tono adecuado y hacerlo en el momento oportuno. Son también conductas no verbales, porque buena parte de la comunicación entre los seres humanos se hace a través de gestos, por la mirada, una sonrisa, una caricia, etc.

En tal sentido, la presente investigación busca identificar estas conductas verbales y no verbales, describirlas y compararlas según el sexo y la especialidad que cursan los estudiantes de la Facultad de Ciencias de la Educación.

\section{OBJETIVOS}

\section{Objetivo General}

Identificar el nivel de habilidades sociales según sexo y especialidad, que presentan los estudiantes de la Facultad de Ciencias de la Educación.

\section{Objetivos Específicos}

1. Describir el nivel de habilidades sociales según sexo y especialidad de los estudiantes de la FACE.

2. Comparar el nivel de habilidades sociales según sexo y la especialidad de estudio

\section{MARCOTEÓRICO}

La habilidad es la capacidad que tiene todo ser humano de realizar o ejecutar algo, se refiere al saber hacer, respondiendo a uno de los pilares de la educación propuestos por el informe de DELORS de la UNESCO (1996).

Con el término de habilidades sociales nos vamos a referir a un amplio número de respuestas, destinadas a establecer y efectuar interacciones sociales. Estas habilidades incluyen la disposición para comunicar con éxito, la cual precisa a su vez, de la aptitud de imaginarse a uno mismo en el papel de la otra persona: comprender su comportamiento y reaccionar ante el mismo con eficacia (García y Nagaz 1998).
Las habilidades sociales son conductas que facilitan la relación interpersonal, de forma no agresiva ni inhibida,sino asertiva. Esas conductas hábiles requieren, previamente, unas capacidades cognitivas (como por ejemplo tener pensamiento alternativo, consecuencial y sobre todo de perspectiva, que significa saber ponerse en el lugar de otro). Igualmente la habilidad social implica control de las propias emociones y algún entrenamiento de la motricidad. Pero, ineludiblemente, las verdaderas habilidades sociales requieren una madurez moral correspondiente a la edad de cada uno, sin ella las habilidades se convierten en manipulación y cinismo (Segura Manuel 2002).

El concepto de habilidades sociales, se viene estudiando actualmente como una habilidad especial que debe de ser fomentada fuertemente y lo más tempranamente posible para el desarrollo de la Inteligencia Emocional, un término difundido mundialmente por el periodista y psicólogo Daniel Goleman, que nos dice que "es la capacidad de sentir, entender, controlar y modificar estados anímicos propios y ajenos".

La inteligencia emocional nos permite tomar conciencia de nuestras emociones, comprender los sentimientos de los demás, tolerar las presiones y frustraciones que soportamos en el trabajo, acentuar nuestra capacidad de trabajar en equipo y adoptar una actitud empática y social, que nos brindará mayores posibilidades de desarrollo personal.

Para Howard Gardner, psicólogo e investigador de la inteligencia, considera que la inteligencia emocional está comprendida en la inteligencia interpersonal e inteligencia intrapersonal. En vez de inteligencia interpersonal también se puede decir inteligencia social, que caracteriza a las personas que desarrollan y modelan habilidades sociales, aunque tiene un segundo sentido: comprometido socialmente, servicial, algo que distingue a las buenas personas. En cambio la inteligencia emocional está significada, objetivamente, que uno puede comprometerse socialmente $y, \sin$ embargo, ser muy antisocial e inhumano.

Entonces la habilidad social es la capacidad de ejecutar una conducta de intercambio con resultados favorables entraña una serie de factores, se refiere a la capacidad de ejecutar la conducta, la conducta en sí, el resultado y lo favorable de esta. La capacidad de ejecutar la conducta supone la posibilidad de realizar una acción, esta acción que se revierte en la posibilidad de construir hechos se traduce en la persona en lo que constantemente llamamos actos. De alguna manera ello implica y considera al ser humano como generador de actos propios o una voluntad de acciones. La acción de la persona se determina en una o varias conductas interpersonales relacionadas entre sí (intercambio), siendo la conducta empleada un medio para alcanzar una meta que intenta resolver el conflicto entre el yo y la circunstancias o viceversa. $\mathrm{Si}$ el resultado o la consecuencia de la conducta obtenida es la conformidad 
del yo y de la circunstancia, entonces se ha resuelto favorablemente el conflicto. La habilidad social debe considerarse dentro de un marco determinado, esto se debe a las marcadas diferencias que establece cada país, donde existen sistemas de comunicación distintivos, que van a tipificar la cultura: los hábitos, costumbres y la propia idiosincrasia crea con el pasar de los años, e influidos por las situaciones externas.

Para abordar el constructo habilidad social es muy importante tener en cuenta la definición de interacción social; la diferencia entre intercambio e interacción estriba en que el primero se refiere a un trueque entre objetos y personas mientras que el segundo se refiere a un acción mutua o de reciprocidad. Dicho de otro modo, la acción se desarrolla entre varias personas, lo cual da la posibilidad de una retroalimentación, así la noción de habilidad social entra en el ámbito de lo reciproco y en el continuo de la acción del uno sobre el otro y de este sobre aquel. La interacción fundamenta la respuesta del otro en una combinación de la acción iniciada; de manera que la habilidad social no termina sin la acción del otro, es decir, la habilidad social supone beneficios para ambos implicados.

El contacto humano, fundamento de las relaciones de intercambio, se produce en un indeterminado caudal de consecuencias de uno para con el otro y viceversa, por lo que existe todo el tiempo (en el momento del intercambio) una constante ida y vuelta. Sin lugar a dudas si cada ida o cada vuelta fuera distinta completamente a una experiencia anterior, entonces las posibilidades de efectos negativos fuera mayor, por lo que se correría el riesgo de no encontrar patrones de conducta más o menos parecidos que posibilitaran la experiencia positiva y disminución del temor al intercambio. Sin embargo, existe un código de intercambio que al igual que el lenguaje, con la utilización de signos, le es posible ejecutar infinidad de expresiones en múltiples ocasiones de la vida. La relación de intercambio no solo se produce favorablemente, sino que existe un número muy inferior al intercambio favorable, de conductas de intercambio desfavorables. Así, la habilidad social puede definirse en virtud del afrontamiento de intercambios desfavorables, de ahi que establecer el contacto sea muy importante, pero en algunos casos es imprescindible saber descontactar.

\section{MATERIAL Y MÉTODOS}

\section{Tipo y Diseño de Investigación}

La presente investigación corresponde al tipo descriptivo, de diseño descriptivo.

\section{Población y Muestra}

Las especialidades de SPRO, LEGE y IETI de la Facultad Ciencias de la Educación son:

\begin{tabular}{|l|l|c|r|}
\hline ESPECIALIDAD & \multicolumn{1}{|c|}{ AÑO } & POBLACIÓN & \multicolumn{1}{c|}{ MUESTRA } \\
\hline SPRO & Primero & 39 & 29 \\
\hline IETI & Primero & 45 & 34 \\
\hline LEGE & Primero & 53 & 39 \\
\hline & TOTAL & 137 & 102 \\
\hline
\end{tabular}

\section{PROCEDIMIENTOS, TÉCNICAS E INSTRUMENTOS DE RECOLECCIÓN DE DATOS}

\section{Procedimiento:}

Personal y de primera fuente.

\section{Técnicas e Instrumentos:}

Técnicas de recolección de datos que utilizamos para la obtención de datos sobre la formación en las actividades de este trabajo de investigación, será la encuesta cuyo instrumento será el cuestionario.

El cuestionario consta de veinte preguntas que buscan explorar las habilidades sociales que se expresaran en los siguientes indicadores:

$\begin{array}{llll}\text { a. Siempre } & 4 & \text { puntos } \\ \text { b. A menudo } & 3 & \text { puntos } \\ \text { c. Muy pocas veces } & 2 & \text { puntos } \\ \text { d. Alguna vez } & 1 & \text { puntos } \\ \text { e. Nunca } & 0 & \text { puntos }\end{array}$

Lo que significa

- Siempre: Habilidades sociales óptimas

- A menudo: Habilidades sociales adecuadas

- Muy pocas veces: Competencias sociales adecuadas mejorables

- Alguna vez: Pobres destrezas de tipo social

- Nunca: Muy pobres destrezas de tipo social

\section{RESULTADOS Y DISCUSIÓN}

Según el Cuadro $\mathrm{N}^{\circ} 4$ podemos observar los resultados en porcentajes del cuestionario aplicado a los estudiantes de las especialidades de SPRO, IETI y LEGE sobre habilidades sociales.

El $61,54 \%, 58,02 \%$ y $4, .15 \%$ de las especialidades SPRO, IETI y LEGE a menudo muestran habilidades sociales, mientras que el $38,46 \%, 30,77 \%$ y $23,53 \%$ de las especialidades SPRO, IETI y LEGE siempre muestran habilidades sociales, un $17,65 \%, 15,38 \%$ y $7,69 \%$ alguna vez lo dan a conocer.

Por lo expuesto, se puede establecer que un porcentaje mayoritario de $61,54 \%, 58,02 \%$ y $46,15 \%$ de las especialidades LEGE, IETI y SPRO a menudo muestran habilidades sociales, se puede evidenciar por los porcentajes presentados, que los estudiantes de la especialidad de LEGE presentan un mayor porcentaje 
en relación con las otras especialidades; sin embargo, también se evidencia que hay un mayor porcentaje $17,65 \%$ de la especialidad de IETI que algunas veces dan a conocer sus habilidades sociales. Cabe anotar que en el indicador de siempre muestran habilidades sociales existen porcentajes próximos con la especialidad de SPRO y de LEGE estando más distante la especialidad de IETI.

El siempre mostrar habilidades sociales en sus relaciones sociales es lo que se deberia encontrar de manera mucho más acentuada, tratándose de que la muestra lo integran jóvenes, que interactúan frecuentemente con el mismo grupo, lo que podría facilitar las expresión de este tipo de conducta.

Cuadro $\mathrm{N}^{\circ} 04$ Habilidades sociales en estudiantes por especialidad de la Facultad Ciencias de la Educación de la $U N J B G$

\begin{tabular}{|c|c|c|c|c|c|c|}
\hline \multirow{3}{*}{ Indicadores } & \multicolumn{6}{|c|}{ Especialidad } \\
\hline & \multicolumn{2}{|c|}{ SPRO } & \multicolumn{2}{|c|}{ IETI } & \multicolumn{2}{|c|}{ LEGE } \\
\hline & $f$ & $\%$ & $\mathrm{~F}$ & $\%$ & $f$ & $\%$ \\
\hline Nunca & 0 & 0.00 & 0 & 0.00 & 0 & 0.00 \\
\hline Muy pocas veces & 0 & 0.00 & 0 & 0.00 & 0 & 0.00 \\
\hline Alguna vez & 6 & 15.38 & 6 & 1765 & 3 & 7.69 \\
\hline A menudo & 18 & 46.15 & 20 & 58.82 & 24 & 61.54 \\
\hline Siempre & 15 & 38.46 & 8 & 23.53 & 12 & 30.77 \\
\hline Total & 29 & 100.00 & 34 & 100.00 & 39 & 100.00 \\
\hline
\end{tabular}

Fuente: Cuestionario de habilidades sociales aplicado a estudiantes de las especialidades de SPRO, IETI Y LEGE de la FACE - UNJBG

Gráfico $\mathrm{N}^{\circ} 04$ Habilidades sociales en estudiantes por especialidad de la Facultad Ciencias de la Educación de la UNJBG.

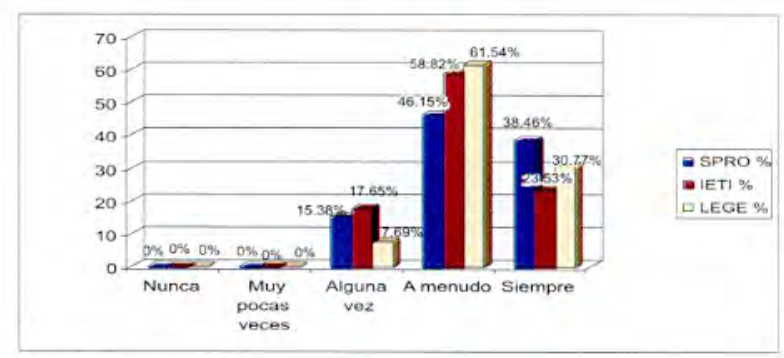

Fuente: Cuadro $N^{\circ} 04$

De acuerdo con el Cuadro $\mathrm{N}^{\circ} 09$, podemos observar los resultados en porcentajes del cuestionario aplicado a los estudiantes según sexo de las especialidades de SPRO, IETI y LEGE de la Facultad de Ciencias de la Educación sobre habilidades sociales.

El $80,00 \%, 55,56 \%$ y $62,50 \%$ de estudiantes varones de las especialidades de SPRO, IETI y LEGE respectivamente a menudo muestran habilidades sociales, mientras que el $10,00 \%, 33,33 \%$ y $25,00 \%$ varones de las especialidad es de SPRO, IETI y LEGE respectivamente siempre muestran habilidades sociales. Finalmente, un $10,00 \%, 11,11 \%$ y $12,50 \%$ varones de las especialidades de SPRO, IETI y LEGE respectivamente alguna vez lo dan a conocer.

El $52,63 \%, 60,00 \%$ y $60,87 \%$ de estudiantes mujeres de las especialidades de SPRO, IETI y LEGE respectivamente a menudo muestran habilidades sociales, mientras que el $21,05 \%, 20,00 \%$ y $34,78 \%$ mujeres de las especialidades de SPRO, IETI y LEGE respectivamente siempre muestran habilidades sociales. Finalmente un $26,32 \%, 20,00 \%$ y $4,35 \%$ mujeres de las especialidades de SPRO, IETI y LEGE respectivamente alguna vez lo dan a conocer.

Por lo expuesto, se puede establecer que un porcentaje mayoritario de el $80,00 \%, 55,56 \%$ y $62,50 \%$ de estudiantes varones de las especialidades de SPRO, IETI y LEGE respectivamente a menudo muestran habilidades sociales respectivamente así como el $52,63 \%, 60,00 \%$ y $60,87 \%$ de estudiantes mujeres de las especialidades de SPRO, IETI y LEGE respectivamente a menudo muestran habilidades sociales. Así mismo podemos notar que los estudiantes varones de la especialidad de SPRO reflejan un porcentaje mayor en comparación con las otras especialidades que se interpreta como mejores habilidades sociales. Pero en el caso de estudiantes mujeres notamos un mayor porcentaje en la especialidad de LEGE, pero cerca de ello a la especialidad de IETI. Por los porcentajes presentados de acuerdo con el sexo por cada especiliad no evidenciamos porcentajes tan marcados en una $u$ otra especialidad que nos hagan suponer diferencias significativas en las habilidades sociales tomando la variable sexo.

Cuadro $\mathrm{N}^{\circ} 09$ Habilidades sociales en estudiantes según sexo, según especialidades de SPRO, IETI y LEGE de la Facultad Ciencias de la Educación de la UNJBG

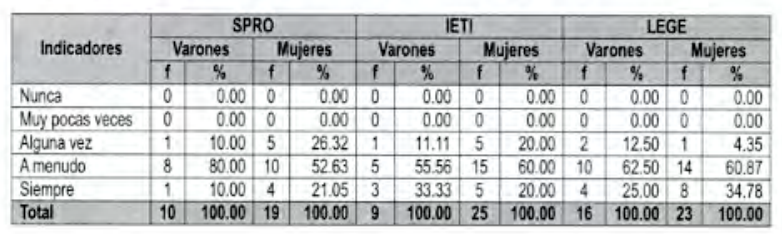

Fuente: Cuestionario de habilidades sociales aplicado a estudiantes de la Facultad de Ciencias de la Educación

Gráfico $\mathrm{N}^{\circ} 09$ Habilidades sociales en estudiantes según sexo, según especialidades de SPRO, IETI y LEGE de la Facultad Ciencias de la Educación de la UNJBG

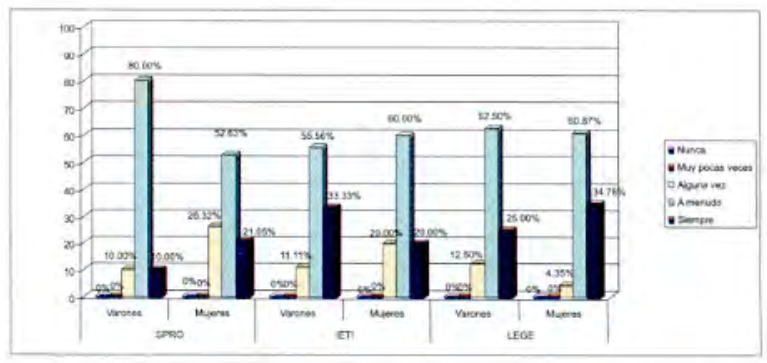

De acuerdo con el cuadro $\mathrm{N}^{\circ} 10$, podemos observar los resultados en porcentajes del cuestionario aplicado a los estudiantes de la Facultad de Ciencias de la Educación según sexo sobre habilidades sociales. 
El $71,43 \%$ y $55,22 \%$ de varones y mujeres respectivamente a menudo muestran habilidades sociales, mientras que el $17,14 \%$ y $28,36 \%$ de varones y mujeres respectivamente siempre muestran habilidades sociales, un $11,43 \%$ y $16,42 \%$ alguna vez lo dan a conocer.

Por lo expuesto, se puede establecer que un porcentaje mayoritario de $71,43 \%$ y $55,22 \%$ de varones y mujeres respectivamente a menudo muestran habilidades sociales, que .si agregamos a ello el $28,36 \%$ y $17,14 \%$ de mujeres y varones respectivamente, que siempre muestran habilidades sociales, concluiremos que las diferencias existentes entres hombres y mujeres en cuanto a habilidades sociales son mínimas pero no significativas.

Cuadro $\mathrm{N}^{\circ} 10$ Habilidades sociales en estudiantes según sexo de la Facultad Ciencias de la Educación

\begin{tabular}{|c|c|c|c|c|}
\hline \multirow{2}{*}{ items } & \multicolumn{2}{|c|}{ Varones } & \multicolumn{2}{|c|}{ Mujeres } \\
\hline & $f$ & $\%$ & $f$ & $\%$ \\
\hline Nunca & 0 & 0.00 & 0 & 0.00 \\
\hline Muy pocas veces & 0 & 0.00 & 0 & 0.00 \\
\hline Alguna vez & 4 & 11.43 & 11 & 16.42 \\
\hline A menudo & 25 & 71.43 & 37 & 55.22 \\
\hline Siempre & 6 & 17.14 & 19 & 28.36 \\
\hline Total & 35 & 100.00 & 67 & 100.00 \\
\hline
\end{tabular}

Fuente: Cuestionario aplicado a estudiantes seguin sexo de la FACE

Gráfico $\mathrm{N}^{\circ} 10$ Habilidades sociales en estudiantes según sexo de la Facultad Ciencias de la Educación

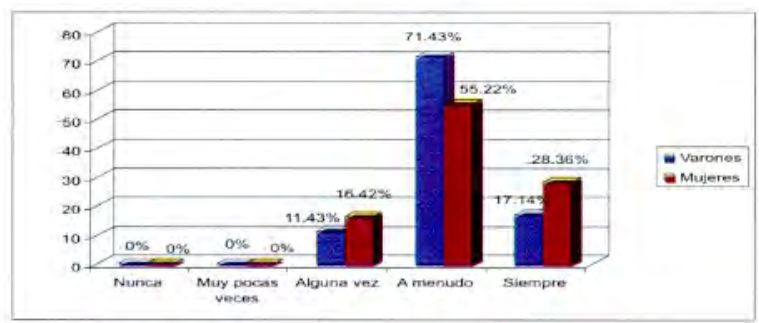

Fuente: Cuadro $N^{\circ} 70$

\section{HIPÓTESIS}

\section{Hipótesis-Específica $\mathrm{N}^{\circ} 01$ :}

"No.existen diferencias significativas en las habilidades sociales según el sexo en los estudiantes de la Facultad de Ciencias de la Educación."

Para contrastar dicha hipótesis nos basamos en el cuestionario aplicado a los estudiantes según su sexo de las especialidades de Ciencias Sociales y Promoción Socio-cultural, Idioma Extranjero, Traductor e Intérprete y Lengua, Literatura y Gestión Educativa de la:Facultad Ciencias de la Educación, según los cuadros $\mathrm{N}^{\circ} \cdot 06,07,08,09$ y 10 del cuestionario aplicado a los estudiantes donde se aprecia que la mayoría de varones y mujeres a menudo muestran habilidades, y sus diferencias no son significativas en las habilidades sociales.

Por lo que podemos decir que la hipótesis especifica $\mathrm{N}^{\circ} 01$ queda comprobada.

Con un riesgo de equivocación menor al 0,001\%,

\section{Hipótesis Específica $\mathrm{N}^{\circ} 02$ :}

"No existen diferencias significativas en las habilidades sociales según la especialidad en los estudiantes de la Facultad de Ciencias de la Educación."

Para contrastar dicha hipótesis nos basamos en el cuestionario aplicado a los estudiantes según su especialidad en Ciencias Sociales y Promoción Sociocultural, Idioma Extranjero, Traductor e Intérprete y Lengua, Literatura y Gestión Educativa de la Facultad Ciencias de la Educación; según los cuadros $\mathrm{N}^{\circ} 01,02$, 03,04 y 05 , a menudo muestran habilidades y sus diferencias entre las especialidades no son significativas en las habilidades sociales de los estudiantes.

Por lo que podemos decir que la hipótesis específica $\mathrm{N}^{\circ} 02$ queda comprobada.

Con un riesgo de equivocación menor al $0.001 \%$.

\section{CONCLUSIONES}

1. No existen diferencias significativas en las habilidades sociales tomando en cuenta la variable sexo en los estudiantes de la muestra de investigación de la Facultad de Ciencias de la Educación.

2. No existen diferencias significativas en las habilidades sociales según la especialidad de SPRO, IETI y LEGE.

\section{RECOMENDACIONES}

1. Siendo importante que los estudiantes aprendan y desarrollen habilidad sociales que les permita comportarse den un modo socialmente eficiente, es necesario que el curriculo considere asignaturas y contenidos que permitan que los estudiantes adquieran habilidades sociales que optimicen sus relaciones interpersonales.

2. La labor del docente es fundamental, su labor no solo debe de centrarse en ser un trasmisor de conocimientos, es necesario que en su actuar pedagógico modele conductas prosociales como: ser empático, asertivo, proactivo, fíder, que le permita generar una experiencia positiva y motivadora en el aula, promoviendo habilidades sociales óptimas.

3. El aprendizaje de habilidades sociales no solo se 
adquiere en la escuela o la universidad de ahi que es tarea y responsabilidad de los padres inculcar desde una temprana edad en sus miembros el cultivo y desarrollo de habilidades sociales, pero, conociendo el nivel sociocultural escaso de un buen porcentaje de padres de familia en nuestro país, es necesario que se contribuya en su formación. Una buena medida podría ser el de impulsar las escuelas para padres que tomen como eje de enseñanza la forma cómo educar y desarrollar las habilidades sociales.

\section{REFERENCIAS BIBLIOGRÁFICAS}

COVEY, Stephen. Los 7 hábitos de la gente altamente efectiva. Editorial Paidos, Buenos Aires, 1996.

CRAIG, Grace J. Desarrollo psicológico. Editorial Prentice Hall Inc, Sexta edición, México, 1992.

ENCICLOPEDIA OCÉANO. El estudiante exitoso. Barcelona.

GOLEMAN, Daniel. La psicología del autoengaño. Editorial Atlántida, Buenos Aires, 1997.

HIDAlgO, Carmen Gloria. Comunicación interpersonal. Ediciones Universidad Católica De Chile, Segunda edición, Chile, 1991.

HIDALGO, Benigno. Cómo desarrollar habilidades y competencias. INADEP, Lima, 1997.

MARINA, José A. Aprender a convivir: Barcelona Ariel, 2006.
MATA MOLINA, F y otros (2000). Emocionalmente inteligente. Revista Calidad.

MEYER, Paul. Pensamientos para una mayor fuerza personal. Editorial Sucess Motivation Institute INC, EE.UU, 1972.

MONTALVA OLIVARES, Doris e HIDALGO MATOS, Menigno(1998). Habilidades personales y sociales del docente. Co-edic.INADEP, EDUPERÚ. Lima.

MONTALVA, Doris. Estrategias para el desarrollo sociopersonal. INIDE, Lima, 1988.

SEGURA MORALES, Manuel. Ser persona $y$ relacionarse: Habilidades cognitivas y sociales $y$ crecimiento moral. Ediciones Narcea, Madrid. 2002.

VARGAS, G. Julia. Técnicas de aprendizaje vivencial para el fortalecimiento de la autoestima y el desarrollo personal, Lima, 1993.

Revista: Aprendiendo habilidades sociales. Colegio de Psicólogos del Perú, Consejo Directivo Regional, Lima.

www.monografías.com/trabajos 15 /inteligenciaemocional/inteligencia-emocional.shtml

www.inteligencia-emocional.org infoarrobainteligencia-emocional.org www.psicología-online.com/ monografias/1/index.shtml. 